\title{
Nanotechnology on Boiling Heat Transfer for a Next-generation Cooling Technology
}

Hyung Hee Cho* and Beom Seok Kim

Department of Mechanical Engineering, Yonsei University, Yonsei-ro, Seodaemun-gu, Seoul, Korea

\begin{abstract}
Nanotechnology has suggested a novel approach for the breakthrough of typical cooling technology especially on boiling heat transfer. As a hot issue in heat transfer fields, the feasible methods based on nanoscale structure creations can be concretized with regard to the compatibility for the control of morphological surface roughness and consequential wettability characteristics. Those two principal factors, which are definitely confirmed to dominate physics on boiling heat transfer, are controlled by nanoscale surface treatment techniques and it finally results in innovative improvements to effectively dissipate thermal-load from hot or high heat generating components according to the roughened and the water-attractive characteristics. Densely distributed nanowires as well as hierarchical morphologies via nano-structures and wettability control techniques on local area will be briefly demonstrated regarding the effectiveness of surface manipulation and the improvement of cooling performances. Progressive research on structural robustness and stability would be required in aspect of reliable performance and life-time of actual applications.
\end{abstract}

Keywords: Nanotechnology; Cooling technology; Boiling heat transfer; Nanostructure; Surface roughness; Surface wettability

Cooling technology, which makes possible to dissipate generated or transmissive heat from hot spots to atmosphere, based on heat transfer is an essential ingredients for practical modern industry fields. For instance, lot of industrial products embodying Central Process Unit (CPU) should be designed to maintain the suitable operating temperature not to exceed critical limits which cause performance degradation or even the component's failure. As the quantity of heat generation rapidly increases according to the increase of the integration density of electric circuits, advanced cooling technologies are required as pre-requisite criteria for thermal designing of devices.

We have looked for sufficient and feasible methods in the aspect of cooling capacity and effectiveness based on heat transfer dynamics. Boiling heat transfer has been considered as a powerful method for high-thermal load dissipations accompanying vigorous phase-change of liquid coolant on a hot spot surface [1,2]. The technique with much higher heat transfer coefficients compared to typical single-phase convective cooling methods like natural and forced convection via gasor liquid-phase coolant, has contributed to the appearance of modern electronic devices with astonishing performances $[3,4]$.

Since the middle of 2000s, nanotechnologies have been magnified as a novel innovative approach to improve the cooling performance of boiling heat transfer [5-7]. Based on thermo-physical fundamentals on boiling heat transfer, we can present two principal factors which dominantly determine the cooling performance; one is surface roughness and the other is wettability characteristics. Herein, the manipulated surface morphology via nanoscale structures, like vertically aligned nanowires [6-10], is able to increase the roughness extremely and intensify the hydrophilicity towards super-wetting regime which is clearly favorable to wetting of the target surface by liquid-phase coolant. At first, higher heat dissipation capacity can be attributed to the increase of surface roughness, which means the extended interfacial contact area between the solid surface and the liquid coolant. Even though silicon nanowires have relatively low thermal conductivity of about $8 \mathrm{~W} / \mathrm{m} \cdot \mathrm{K}$ compared to bulk silicon substrate of about 140 $\mathrm{W} / \mathrm{m} \cdot \mathrm{K}[11]$, the heat dissipation capability under boiling conditions was considerably improved. This can be demonstrated by other aspects on the morphological change via the structures. Surface morphology in company with nano/microscale vacant area formed by the coalescence of distributed nano-structures can play a role as a structural catalyst for the vaporizing of the coolant. The other factor, surface wettability, has also been verified that hydrophilic characteristics of the surfaces result in the enhancement of surface re-wetting properties and then it helps to increase Critical Heat Flux (CHF) to extend maximum heat dissipation capacity. The effects of the surface roughness and the wettability on boiling performances are schematically described in Figure 1 according to the variation of typical boiling curves.

As a simple method via metal assisted chemical etching of silicon, a superhydrophilic surface inducing rapid hemi-wicking of liquid water can be obtained maintaining extremely rough surface with boilingfavorable cavity structures [10]. Moreover, we can convert the local contact angle from superhydrophilic to superhydrophobic regime over 160 degrees by manipulating surface free energy of the substrate [8]. The simple and cost-effective methods based on the nanotechnology have been adopted to characterize boiling performances on flow boiling as well as pool boiling conditions.

Even though there are still undetermined demonstrations on the morphological contribution of the embodied structures, typical performance indexes like CHF, heat transfer coefficient, onset of nucleate boiling and so on, have been greatly enhanced

*Corresponding author: Hyung Hee Cho, Department of Mechanical Engineering, Yonsei University, Yonsei-ro, Seodaemun-gu, Seoul, Korea, Tel.: +82 22123 2828; Fax: +82 2312 2159; E-mail: hhcho@yonsei.ac.kr

Received August 25, 2012; Accepted August 27, 2012; Published September 03, 2012

Citation: Cho HH, Kim BS (2012) Nanotechnology on Boiling Heat Transfer for a Next-generation Cooling Technology. J Material Sci Eng 1:e106. doi:10.4172/2169 0022.1000e106

Copyright: (c) $2012 \mathrm{Cho} \mathrm{HH}$, et al. This is an open-access article distributed under the terms of the Creative Commons Attribution License, which permits unrestricted use, distribution, and reproduction in any medium, provided the original author and source are credited. 
Citation: Cho HH, Kim BS (2012) Nanotechnology on Boiling Heat Transfer for a Next-generation Cooling Technology. J Material Sci Eng 1:e106. doi:10.4172/2169-0022.1000e106

Page 2 of 2

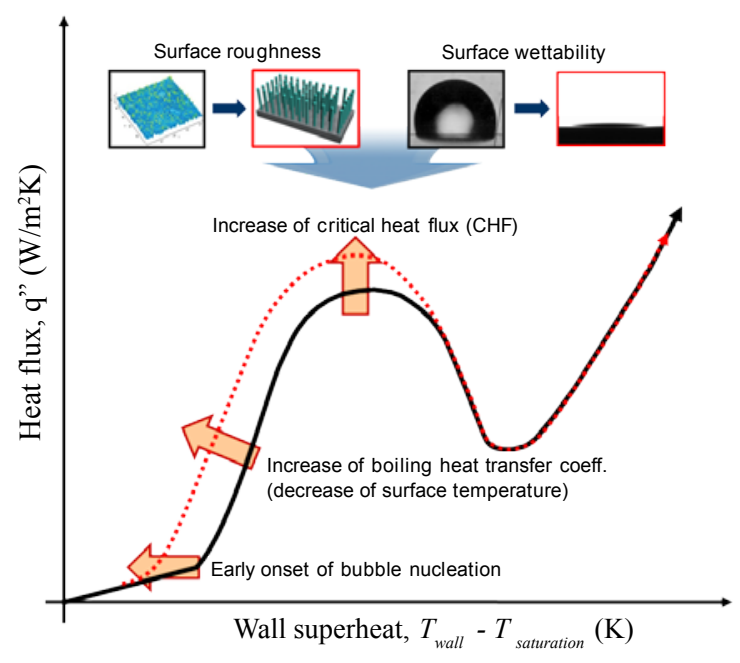

Figure 1: Typical boiling curves and performance evolution according to the manipulation of surface roughness and wettability. A boiling favorable surface satisfying the conditions, those are extended surface area, structural catalyst for beneficial nucleation and intensified re-wetting properties, could improve the heat transfer performances in the aspects of less-required thermal load for $\mathrm{ONB}$, and increase of heat transfer coefficient and CHF.

indicating fascinating advances in heat transfer fields. Even though nanotechnologies suggested a new way for the breakthrough of technical limits on boiling heat transfer, further progressive researches in aspects of structural reliability, robustness and long-term stability under an exposed environment would be required for the actual realization on products.

\section{References}

1. Wu SJ, Shin $\mathrm{CH}$, Kim KM, Cho HH (2007) Single-phase convection and boiling heat transfer: Confined single and array-circular impinging jets. International journal of multiphase flow 33: 1271-1283.

2. Shin $\mathrm{CH}$, Kim KM, Lim SH, Cho HH (2009) Influences of nozzle-plate spacing on boiling heat transfer of confined planar dielectric liquid impinging jet. Int $\mathrm{J}$ Heat Mass Transf 52: 5293-5301.

3. Goldstein RJ, Ibele WE, Patankar SV, Simon TW, Kuehn TH, et al. (2006) Heat transfer - A review of 2003 literature. Int J Heat Mass Tran 49: 451-534.

4. Garimella SV, Fleischer AS, Murthy JY, Keshavarzi A, Prasher R, et al. (2008) Thermal Challenges in Next-Generation Electronic Systems. IEEE Transactions on Components and Packaging Technologies 31: 801-815.

5. Li C, Wang Z, Wang PI, Peles Y, Koratkar N, et al. (2008) Nanostructured copper interfaces for enhanced boiling. Small 4: 1084-1088.

6. Chen R, Lu MC, Srinivasan V, Wang Z, Cho HH, et al. (2009) Nanowires for Enhanced Boiling Heat Transfer. Nano Lett 9: 548-553.

7. Li D, Wu GS, Wang W, Wang YD, Liu D, et al. (2012) Enhancing Flow Boiling Heat Transfer in Microchannels for Thermal Management with MonolithicallyIntegrated Silicon Nanowires. Nano Lett 12: 3385-3390.

8. Kim BS, Shin S, Shin SJ, Kim KM, Cho HH (2011) Control of Superhydrophilicity/ Superhydrophobicity using Silicon Nanowires via Electroless Etching Method and Fluorine Carbon Coatings. Langmuir 27: 10148-10156.

9. Shin S, Kim BS, Kim KM, Kong BH, Cho HK, et al. (2011) Tuning the morphology of copper nanowires by controlling the growth processes in electrodeposition. $J$ Mater Chem 21: 17967-17971.

10. Kim BS, Shin S, Shin SJ, Kim KM, Cho HH (2011) Micro-nano hybrid structures with manipulated wettability using a two-step silicon etching on a large area. Nanoscale Res Lett 6: 333.

11. Hochbaum Al, Chen R, Delgado RD, Liang W, Garnett EC, et al. (2008) Enhanced thermoelectric performance of rough silicon nanowires. Nature 451 163-167. 